\title{
Marfi Tokalz A correction to my paper "Connections between some notions of completeness of structural propositional calculi"
}

There is a mistake in my paper "Connections between some notions of completeness of structural propositional calculi", Studia Logica XXXII (1973), namely the definition of inconsistency of a logic (p. $78_{5}$ ) is not correct. The correct definition is as follows:

The consequences which satisfy the condition $C n(X)=S$ for every $X \subseteq S$ will be called inconsistent.

In fact, my definition in the paper mentioned just constitutes a definition of a trivial consequence. It is defined the following way:

A consequence $C n$ is called trivial provided for every $\alpha \in S, C n(\alpha)=S$.

According to the above remarks, one more fragment of the paper "Connections..." needs changing. The phrase "no consistent structural calculus would be Post-complete", p. $80^{14}$, has to be replaced by the following: "no non-trivial structural calculus would be Post-complete".

I am very indebted to Professor David Makinson who called attention to this error. 